\title{
EVALUACIÓN DEL AYUNTAMIENTO MUNICIPAL MEDIANTE UNA ENCUESTA DE OPINIÓN EN REPÚBLICA DOMINICANA. EL CASO DEL MUNICIPIO DE BAITOA EN LA PROVINCIA DE SANTIAGO The case study of the municipality of Baitoa, a province of santiago, Dominican Republic. An evaluation of the city council by means of an opinión survey
}

\section{Leonardo Pérez}

Junta Central Electoral: Santo Domingo, Encargado General de la Junta Municipal de Baitoa, República Dominicana. ORCID: 0000-0001-5965-5253

Correo-e: leonardo.perez1212@gmail.com

\author{
Marco Antonio Rojo Gutiérrez \\ Profesor de Tiempo Completo, Universidad Interna- \\ cional Iberoamericana (UNINI), México \\ ORCID: 0000-0003-4862-8780 \\ Correo-e: marco.rojo@unini.edu.mx
}

\section{Recibido: 15/6/2020 • Aprobado: 26/7/2020}

Cómo citar: Pérez, L., \& Rojo Gutiérrez, M. A. (2020). Evaluación del ayuntamiento municipal mediante una encuesta de opinión en República Dominicana. El caso del municipio de Baitoa en la provincia de Santiago. Ciencia y Sociedad, 45(4), 89-104. Doi: https:// doi.org/10.22206/cys.2020.v45i4.pp89-104

\section{Resumen}

Evaluar los municipios mediante encuestas de opinión es un acto cívico de toda democracia. La encuesta como instrumento permite recolectar información que da cuenta sobre la percepción de los munícipes con relación al desempeño de sus autoridades. Este artículo se fundamenta en una encuesta de opinión aplicada al Ayuntamiento Municipal Baitoa en República Dominicana, busca aportar un diagnóstico que mejore su gestión tomando como base la valoración de sus munícipes. La encuesta está integrada por siete ítems, se aplica de forma presencial a una muestra aleatoria de 260 ciudadanos habitantes del municipio, según el método Bonilla (1993). La investigación beneficia a las autoridades electas para el periodo 20162020. El estudio encuentra que el $69 \%$ de los encuestados percibió de manera buena o excelente la gestión municipal actual, donde de cada diez (10) ciudadanos, seis (6) entienden que la mayor fortaleza de la administración fue brindar servicios básicos (recogida de basura, entre otros). Destaca que la población entiende que la administración informa bien y maneja bien sus recursos en un $41 \%$ de la muestra y que un $34 \%$ valora de forma especial la reparación de las vías de comunicación. Un 51 \% de los encuestados tiene expectativas de que la gestión podría tener una

\begin{abstract}
Evaluating municipalities through opinion polls is a civic act of every democracy. The survey as an instrument allows to collect information that accounts for the perception of the municipalities in relation to the performance of their authorities. This article is based on an opinion survey applied to the Baitoa Municipal Council in the Dominican Republic, seeks to provide a diagnosis that improves its management based on the assessment of its municipalities. The survey is made up of seven items, it is applied in person to a random sample of 260 citizens living in the municipality, according to the Bonilla method (1993). The investigation benefits the elected authorities for the period 2016-2020. The study finds that $69 \%$ of those surveyed perceived the current municipal management as good or excellent, where out of every 10 citizens, 6 understand that the greatest strength of the administration was to provide basic services (garbage collection, among others). It stands out that the population understands that the administration reports well and manages its resources well in $41 \%$ of the sample and that $34 \%$ especially value the repair of communication routes. $51 \%$ of those surveyed have expectations that the management could have a continuous improvement in its
\end{abstract}


mejora continua en su gestión. Un 39 \% desearía que la administración municipal realizara en términos generales más obras municipales, de manera particular, un $44 \%$ de los encuestados desearía que se privilegiara la reparación de vías de comunicación.

Palabras clave: municipio; gobierno municipal; gobernanza; Baitoa; encuesta de opinión.

\section{Introducción}

Evaluar al gobierno es fundamental en una democracia. Esta investigación contribuye en esta dirección con una evaluación al Ayuntamiento Municipal Baitoa de la República Dominicana por medio de una encuesta de opinión. La encuesta de opinión permite conocer la percepción que tienen los munícipes de sus autoridades. Esta encuesta busca mejorar la administración en el manejo de los recursos del Ayuntamiento Baitoa y la imagen que se tiene del gobierno municipal a partir de los resultados obtenidos en los dos años que van de su gobierno.

Se tiene como instrumento de recolección de información la encuesta de opinión que se aplica a una muestra de 260 ciudadanos de un universo de 17,778 habitantes que conforman el municipio, según el método de Bonilla (1993). Se realiza un trabajo de campo para la aplicación de un cuestionario presencial compuesto por siete preguntas, cinco de las cuales son de tipo cerradas y las otras dos preguntas restantes de tipo abiertas. Es la primera investigación de este tipo en Baitoa, no se tiene conocimiento de alguna otra en su tipo, esto permite sentar las bases para futuras investigaciones que apoyen este ejercicio democrático en República Dominicana.

La valoración de las autoridades por parte de la población es fundamental para el funcionamiento del gobierno. Una valoración positiva facilita la efectividad en las acciones o actividades que se realicen management. 39\% would like the municipal administration to carry out more municipal works in general terms, in particular, $44 \%$ of those surveyed would like the repair of communication routes to be privileged.

Keywords: Municipality; municipal government; governance; Baitoa; opinion poll.

a favor de la ciudadanía. Un buen gobierno va a acompañado de una buena percepción. La encuesta de opinión sirve como un instrumento para medir la percepción que se tiene de quienes gobiernan, de ahí la importancia de tener un gobierno transparente y honesto.

A distintos niveles de gobierno, la aplicación de la encuesta de opinión es de utilidad para percibir la sensibilidad que tienen los ciudadanos con relación a las actividades realizadas por el gobierno y la imagen que este proyecta a la ciudadanía, de ahí que también la encuesta de opinión refleje el grado de información que fluye entre gobernantes y gobernados, si este flujo de información no se $\mathrm{da}$, se suele abrir una brecha que afecta a la comunidad en su conjunto, es muy difícil gobernar, por ejemplo, en un municipio donde haya incredulidad o desconfianza.

En esta investigación, las respuestas de percepción reflejan la confianza en la administración y la valoración que se tiene de los servicios básicos que ofrece la alcaldía a pesar de la limitación de recursos económicos para su ejecución. Se destaca, por ejemplo, que las comunidades rurales valoran y demandan mejoras en las vías de comunicación terrestre. En comunidades rurales es recurrente que los caminos no pavimentados sean afectados por las lluvias del trópico y las crecidas de arroyos y cañadas, dificultando así el desplazamiento por las mismas y constituyéndose su reparación en un elemento que dignifica la vida de los comunitarios de esos sectores. 


\section{Evaluación del ayuntamiento municipal mediante una encuesta de opinión en República Dominicana. \\ El caso del municipio de Baitoa en la provincia de Santiago}

Dado los resultados de esta investigación, se recomienda a las autoridades municipales del municipio Baitoa, que para los dos últimos años de su gestión municipal se involucre con mayor fortaleza en la actividades sociales, políticas y comunitarias que buscan la manera de dar respuesta a la necesidad de abastecimiento de agua potable de los munícipes de Baitoa, entendiendo que esta es una de las dos mayores demandas sociales de sus gobernados, así como también la construcción o acondicionamiento, donde lo amerite, de los caminos y carreteras del municipio, la pavimentación de los caminos a secciones, y las carreteras troncales que aún mantienen superficie de tierra o con avanzado estado de deterioro deben ser reparadas o construidas con grado de urgencia, puesto que esta necesidad junto a la demanda de agua potable, constituyen las demandas sociales que mayor impacto tiene su población.

Se subraya la idea de aunar esfuerzos con la sociedad civil y organizaciones sociales, religiosas y políticas de la comunidad para gestionar y demandar de forma conjunta ante el gobierno central de la República Dominicana los recursos estatales para resolver estas históricas problemáticas o para también trabajar con proyectos comunitarios conjuntos que puedan dar respuestas por etapas y sectores a esta problemática.

El documento está dividido en cuatro secciones adicionales a la introducción. En la primera sección se presentan los antecedentes de la investigación, en esta se contextualiza y justifica la pertinencia de la misma, tanto empírica como teóricamente. En la segunda sección se presenta el material y los métodos empleados para llevar a cabo la encuesta de opinión. En la tercera sección se presentan los resultados y, finalmente, en la cuarta sección se exponen las principales conclusiones de nuestro estudio. Al final, el documento se acompaña de la bibliografía de consulta que sirvió como fuente para la elaboración del mismo.

\section{Antecedentes}

Un buen gobierno a nivel nacional, debe sustentarse en la buena gobernanza a nivel municipal, el gobierno municipal es el brazo administrativo más próximo que tiene el cuidado, y es el eje fundamental, de los servicios que demanda la vida en comunidad de cualquier sociedad del mundo. Hay una correspondencia entre un buen gobierno, el crecimiento económico y el incremento del nivel de bienestar. En un buen gobierno hay una mayor participación democrática, una mayor intervención y demanda ciudadana, un mayor flujo de conocimiento y de información que favorece la confianza de los gobernados hacia sus gobernantes (CantoSáenz, 2017; Serrano-Rodríguez, 2015).

En muchos países de Latinoamérica los municipios se encuentran en el proceso de avanzar hacia la apropiada gobernanza, en la República Dominicana se han creado 158 municipios buscando descentralizar el accionar del gobierno; sin embargo, la población aún se encuentra en un proceso de distanciamiento y desconocimiento de los deberes y derechos fundamentales que asisten a los gobernantes y los gobernados, lo que dificulta el sano crecimiento sostenible de esta importante estructura de desarrollo sostenible (Candia-Falcón, 2017; Orduña-Trujillo, 2006; González-Murillo, 2003).

Se busca responder interrogantes comunes de distintas sociedades organizadas bajo la organización de municipios; en esta investigación se pudiera poner en evidencia la debilidad institucional de los gobiernos locales (falta de planificación, escasez de recursos humanos y económicos, escasa transparencia, debilidad en el fluir de las informaciones y rendición de cuenta, etc.) (Gómez-MacFarland, 2017; Böhm, Márquez, Poblette, Repossi y Reta, 2007; SAGARPA-INCA Rural, 2004).

Un interés puntual de la investigación es el aporte al sano y sostenible desarrollo del gobierno municipal de Baitoa para el beneficio de sus ciudadanos, 
concomitantemente con las recomendaciones generales que puedan aplicarse en otros municipios con semejante nivel de desarrollo en el país de República Dominicana. Debido al análisis de la relación entre las variables que surgen de la encuesta de opinión sobre la buena o mala percepción de buenos o malos gobiernos municipales, se identifica un patrón de comportamiento que puede replicarse en otras localidades y que podría dar pie a una agenda de investigación futura sobre el buen gobierno González-Ibarra, Tapia-Vega y Apolinar Valencia, 2017; Molina-Molina y Mayor-Balsas, 2018).

En la República Dominicana, al parecer, no se han encontrado estudios sobre el tema, es decir, encuestas de opinión en municipios rurales con estas características particulares de localidades muy pequeñas del sector rural y con altos niveles de pobreza. Por el contrario, a nivel mundial se tiene evidencia de estudios de opinión, tanto en países en desarrollo como países desarrollados, algunos logrando mayor impacto y cobertura que otros por las condiciones vinculadas al grado de interés colectivo que generen los procesos que se estudian (Kuschick, 2006; Mañas-Ramírez, 2005; Kavanagh, 1994; Abreu-Sojo, 1999; Sunkel, 1989).

Los torneos electorales y los estudios de opinión de mercado son de los estudios de opinión más populares. Los estudios de opinión además del tipo político, tienen un carácter social, económico y humano (Kurschick, 2004). Sin embargo, a nivel de ayuntamiento, por ejemplo, se suele implementar una encuesta de opinión buscando consigo mejorar la calidad de los servicios que se ofrecen a la población residente y visitante de la localidad.

En municipios pequeños y rurales resalta la labor realizada por el Pueblo de los Estados Libres de América (USAID por sus siglas en inglés) con su herramienta investigativa Barómetro de las Américas, en su cuestionario aplicado a la República Dominicana y otros países Latinoamericanos entre los años 2018 y 2019. En este cuestionario se consultó cuál es el problema más grave que está afectando al país, las respuestas con mayor índice de repetición fueron: los políticos y la impunidad (Luna y Soifer, 2015; Cabrera, 2001).

Determinar la percepción de los munícipes sobre sus autoridades es un ejercicio que abona tanto a la democracia representativa como a la democracia participativa. La aplicación de encuestas de opinión permite recopilar información para la toma de decisiones políticas y atender con ello las demandas ciudadanas. La interacción entre gobernantes y gobernados es necesaria y fundamental, incluso como un acto cívico-democrático. Es la interacción del gobierno y la sociedad organizada en colectivos y asociaciones civiles lo que da paso a la transformación de regímenes de gobierno autoritarios a gobiernos democráticos, es decir, hay evidencia que muestra que esta interacción relaja el control político y sirve de contrapeso, por lo que se incentiva a una mayor participación ciudadana en estos actos (Sorribas y Garay-Reyna, 2014; Schneider, 2011; Peter, Fieldhouse y Liu, 2011).

La demarcación rural Baitoa, de la provincia Santiago en la República Dominicana, fue elevada a categoría de municipio el día 10 de mayo del año 2013, mediante una ordenanza del Congreso dominicano (Ley 69-13, 2013). Las elecciones del año 2016 representaron una oportunidad para que las autoridades electas para ese periodo establecieran pautas institucionales para el sano y fuerte crecimiento del nuevo municipio con una visión de futuro para el establecimiento de un plan de gobierno municipal estable, que orbitara entre las expectativas de trabajo mancomunado entre autoridades y sus habitantes.

Al inicio de gobierno se percibía una disyuntiva en la percepción de la población sobre las autoridades electas en el Ayuntamiento Baitoa, es decir, no existía un consenso en torno al buen o mal funcionamiento del ayuntamiento, algunos consideraban 


\section{Evaluación del ayuntamiento municipal mediante una encuesta de opinión en República Dominicana. \\ El caso del municipio de Baitoa en la provincia de Santiago}

que la administración estaba realizando una buena labor, mientras que otros aseguraban que la misma operaba de forma ineficiente, que no realizaba su gestión con la transparencia, ni con la consistencia que debiera hacerlo una buena administración municipal, lo cual generó una controversia en muchas de las comunidades que conforman este municipio.

En República Dominicana la decepción y la molestia pública sobre las autoridades municipales ha ido en aumento. Al parecer, hay un descontento social por actos de corrupción que refleja una crisis severa a nivel institucional al interior del gobierno. Los actos de corrupción se encuentran documentados y tienen una característica particular que no han sido resueltos, es decir, continúan bajo el amparo de la impunidad, la falta de justicia y el debilitamiento del estado de derecho en el país. Estos factores no favorecen la aplicación de políticas públicas municipales incluyentes debido a que se favorecen las necesidades particulares de aquellos afines a los gobernantes por su "color" político (Jiménez-Polanco, 2016).

El sector público requiere de una mejora continua, con el propósito de ir aprendiendo de sus fallas y experimentar mejores procesos y procedimientos a fin de operar con una mayor transparencia y erradicar en lo posible los actos de corrupción. En cada una de las dependencias se requiere de una mayor dirección y liderazgo que privilegie la atención al ciudadano por sobre intereses personales o de partido, en una democracia real, el servidor público no es discrecional, sino que adopta una filosofía de atención a la ciudadanía en general, sin colores ni partidos (Gimeno-Feliu, 2019; Gutiérrez, 2018).

\section{Material y métodos}

Esta investigación busca contribuir a tener un buen gobierno municipal con una buena percepción por parte de sus ciudadanos. Se mide la percepción y se evalúa el desempeño del gobierno municipal de Baitoa. Se utiliza la encuesta de opinión como instrumento para el levantamiento de la información, validando cualitativamente su pertinencia con el objetivo de estudio.

Baitoa es un municipio ubicado en la provincia de Santiago en la región norte de La República Dominicana, con una población aproximada de 17,778 habitantes, este municipio está compuesto por siete secciones políticas territoriales en las cuales se aplica la encuesta. Se toma como muestra de estudio a 260 habitantes de manera aleatoria según el método de Bonilla (1993). Se aplican cuestionarios presenciales de siete (7) preguntas cinco (5) de tipo cerradas y dos (2) de tipo abiertas) (Casas-Anguita, Labradora y Campos, 2003; Martínez de Luna, 2008).

La encuesta en sí misma se presenta como un instrumento ampliamente utilizado debido a la rapidez y a la eficacia en la obtención de información de los miembros de una comunidad que es de interés para el investigador (Fernández-Ruiz, 2015). En los ítems se busca saber cómo la ciudadanía valora las autoridades electas en el periodo 2016-2020, para identificar la percepción que se ha creado de los gobernantes por parte de sus munícipes en sus primeros dos años de gestión e identificar cuáles aspectos de la gobernanza municipal son más altamente valorados, así como fundamentar algunas recomendaciones que pudieran modificar la percepción actual de los ciudadanos.

Se emplea una metodología de tipo mixta debido a que se va a estudiar una variable subjetiva que es la valoración sobre la gestión del gobierno municipal, además de la revisión de documentación y de archivos municipales, también se trabaja con datos estadísticos para la realización del estudio de percepción (Hernández, Fernández y Baptista, 2014). En torno a la parte científica con relación a los ítems, estos fueron validados por opinión de expertos y se seleccionaron aquellas preguntas que permiten identificar de forma más precisa la percepción que se ha creado de los munícipes en relación a la gestión de sus gobernantes. 


\section{Resultados}

Los resultados parten de un cuestionario distribuido y suministrado de manera equitativa entre las muestras. La muestra a su vez fue subdividida de forma calculada entre las distintas secciones del municipio Baitoa y aplicada entre los días 30-31 del mes de mayo del 2019. Con el objetivo de determinar la percepción general de los ciudadanos del Municipio Baitoa sobre los dos primeros ańos de administración de la actual gestión municipal, comprendida entre los años 2016-2020 y dividida por las secciones territoriales que conforman el municipio, entendiendo que esta información es fundamental para interpretar las subsecuentes preguntas de la encuesta se consultó lo siguiente:

Tabla 1. Resultados por sección territorial de como consideran los encuestados los dos primeros años de la actual gestión municipal de Baitoa

\begin{tabular}{|c|c|c|c|c|c|c|c|c|c|}
\hline \multicolumn{7}{|c|}{ Pregunta: ¿Cómo consideras los dos primeros años de la actual gestión municipal de Baitoa? } \\
\hline Alternativas/Sección & Lima & Baitoa & Mocan & San José & López & Ciruelos & Jagua & TOTAL & Pct. \\
\hline Pésima & 8 & 20 & 10 & 0 & 3 & 0 & 0 & 41 & $15.769 \%$ \\
\hline Mala & 5 & 8 & 13 & 9 & 0 & 3 & 3 & 41 & $15.769 \%$ \\
\hline Buena & 3 & 32 & 10 & 35 & 14 & 27 & 32 & 153 & $58.846 \%$ \\
\hline Excelente & 0 & 10 & 6 & 0 & 3 & 0 & 6 & 25 & $9.615 \%$ \\
\hline TOTAL & $\mathbf{1 6}$ & $\mathbf{7 0}$ & $\mathbf{3 9}$ & $\mathbf{4 4}$ & $\mathbf{2 0}$ & $\mathbf{3 0}$ & $\mathbf{4 1}$ & 260 & $100 \%$ \\
\hline
\end{tabular}

Fuente: resultados alcanzados en los cuestionarios de este estudio.

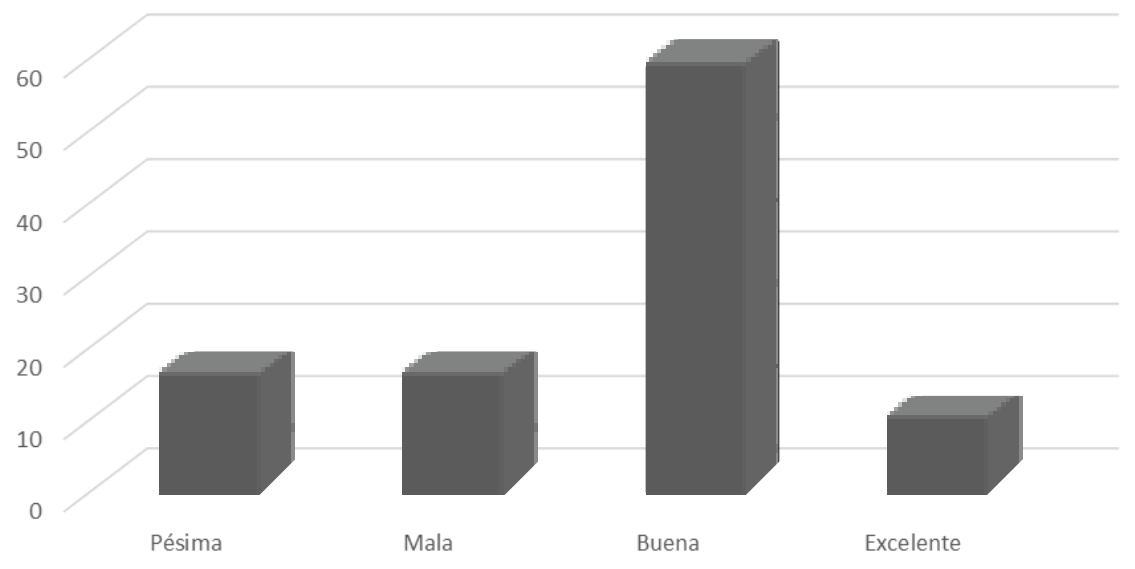

Figura 1. Porcentajes. Cómo consideran los dos primeros años de la actual gestión municipal de Baitoa Fuente: tabla 1 de resultados alcanzados en la pregunta número 1 de este estudio.

$\mathrm{Al}$ analizar las respuestas se puede observar que un sesenta y nueve por ciento $(69 \%)$ de la población del municipio Baitoa valora de forma positiva la actual gestión municipal, un diez por ciento (10
\%) incluso la coloca como excelente administración, lo que demuestra que existe una percepción muy buena en sentido general de las ejecutorias realizadas por la alcaldía en los dos primeros años 


\section{Evaluación del ayuntamiento municipal mediante una encuesta de opinión en República Dominicana. \\ El caso del municipio de Baitoa en la provincia de Santiago}

de esta gestión municipal. En comunidades como La Jagua y Los Ciruelos de donde es oriundo el actual alcalde del Ayuntamiento Baitoa su valoración es mucho mayor en términos porcentuales que en el área más rurales como lo es Mocan o en la zona más distante de su lugar de nacimiento: La Lima; aquí los moradores presentan los índices más bajos de valoración a la actual gestión, lo que podría estar vinculado a una línea de inversión municipal menor o presencia representativa inferior que en las que tiene alta valoración como La Jagua.

La percepción de la población sobre la buena administración municipal, en sentido general, parte de la buena percepción o no que se tenga de quien ocupa la posición de alcalde. Por ello, una de las razones que proyecta una baja valoración de la alcaldía en estas comunidades antes mencionadas podría ser que la imagen del alcalde no ha sido proyectada con la misma intensidad que en otras comunidades en las que conocen su vida social y política desde antes de ocupar la alcaldía y con las que pudiera estar más comprometido a nivel político, o que la administración maneja de forma diferente los servicios e inversión de manera focalizada en estas secciones del municipio. Es interesante cómo en el centro urbano del Municipio de Baitoa y La Jagua casi un quince por ciento (15\%) valora como excelente la gestión municipal actual en los primeros dos ańos, siendo estos dos sectores los de mayor población en todo el municipio y es premisamente donde presentan los picos de valoración excelente más elevados de las siete (7) secciones políticas territoriales del municipio Baitoa.

En sentido general, la valoración de los dos primeros años de la actual gestión municipal es positiva mayoritariamente, por lo que resulta interesante observar cuáles son los aspectos que más valoran los moradores del municipio, con especial énfasis en los sectores con mayor tasa de valoración a la excelencia en la gestión. Asimismo, es importante saber qué es lo que más se entiende debe mejorar la actual gestión municipal en secciones como Mocan, donde valoran negativamente la gestión municipal en un cincuenta y ocho por ciento (58 $\%)$, en franca oposición a los resultados generales de todo el municipio.

Con el objetivo de reflejar cuáles son las ejecutorias que realiza la administración que la población no valora de la mejor manera, se cuestiona sobre: ¿Qué es lo que entiendes que más debe mejorar la actual administración municipal?, esta pregunta se efectúa con el propósito de identificar cuáles son las acciones o políticas públicas más débiles que realiza la administración.

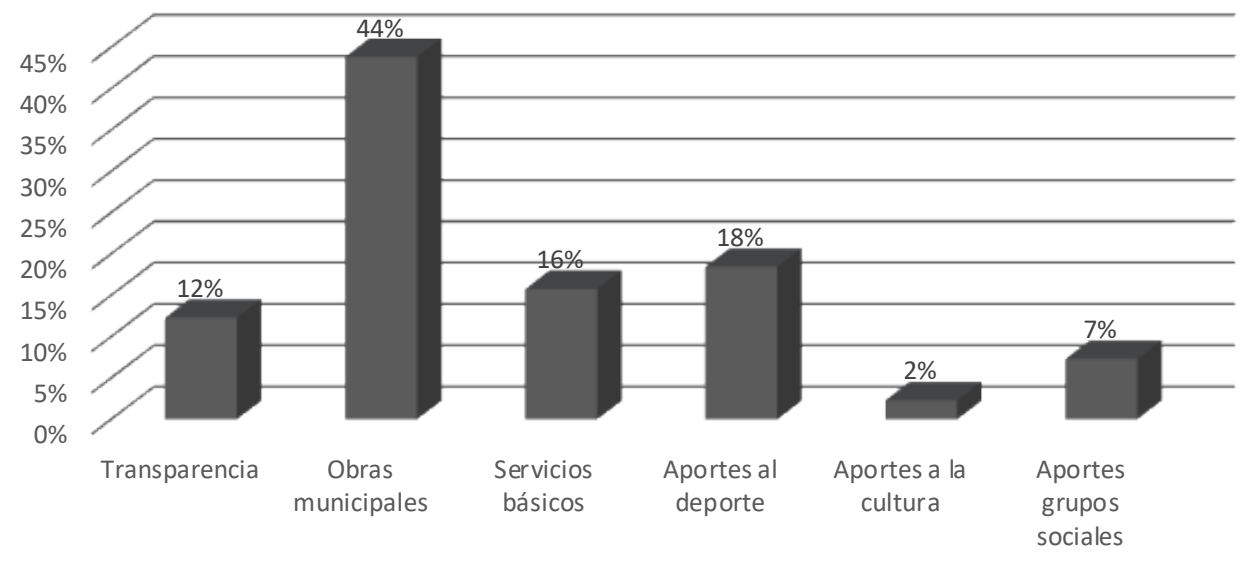

Figura 2. Porcentajes. Lo que más debe mejorar la actual administración municipal

Fuente: tabla 2 de resultados alcanzados en la pregunta número 2 de este estudio. 
Según las respuestas, la población entiende que la alcaldía debe realizar más obras municipales, las cuales dependen directamente del presupuesto que recibe la alcaldía del gobierno central y de la colecta de arbitrios. En las entrevistas se hizo mención de lo bajo que resultan las partidas presupuestarias para dar respuestas a las necesidades de los moradores del municipio. De forma decidida e imponentemente mayoritaria la población entiende que la construcción de obras municipales, entre las que podrían encontrarse canchas, iglesias, aceras, reparación de tramos de carreteras rurales u obras como centros comunales, entre otras, que por tradición en la cultura popular dominicana están vinculadas a las acciones que realizan los ayuntamientos en los municipios, deben tener más presencia la ejecución de las mismas.

El cuarenta y cuatro por ciento ( $44 \%$ ) de la población expresa que desea que la administración municipal de Baitoa realice más obras municipales, dentro de este porcentaje se refleja con mayor notoriedad que la población que se podría considerar más urbana del municipio Baitoa es la que presenta mayores índices de esta observación.

En la comunidad de Mocan, la alcaldía refleja la tasa más baja de valoración con un cincuenta y ocho por ciento (58\%), esto evidencia que además de las pocas obras municipales como mayor debilidad de la administración, los aportes al deporte también deben mejorar de acuerdo con lo medido, siendo estos dos elementos de una constante en los resultados que deben mejorar en los sectores donde se presenta menor valoración de la gestión actual.

Con el objetivo de determinar cuáles son las ejecutorias que realiza la administración que tienen mayor valoración en la población de la que administran sus recursos desde la alcaldía, se preguntó: ¿Qué es lo que entiendes que mejor realiza la actual administración municipal?

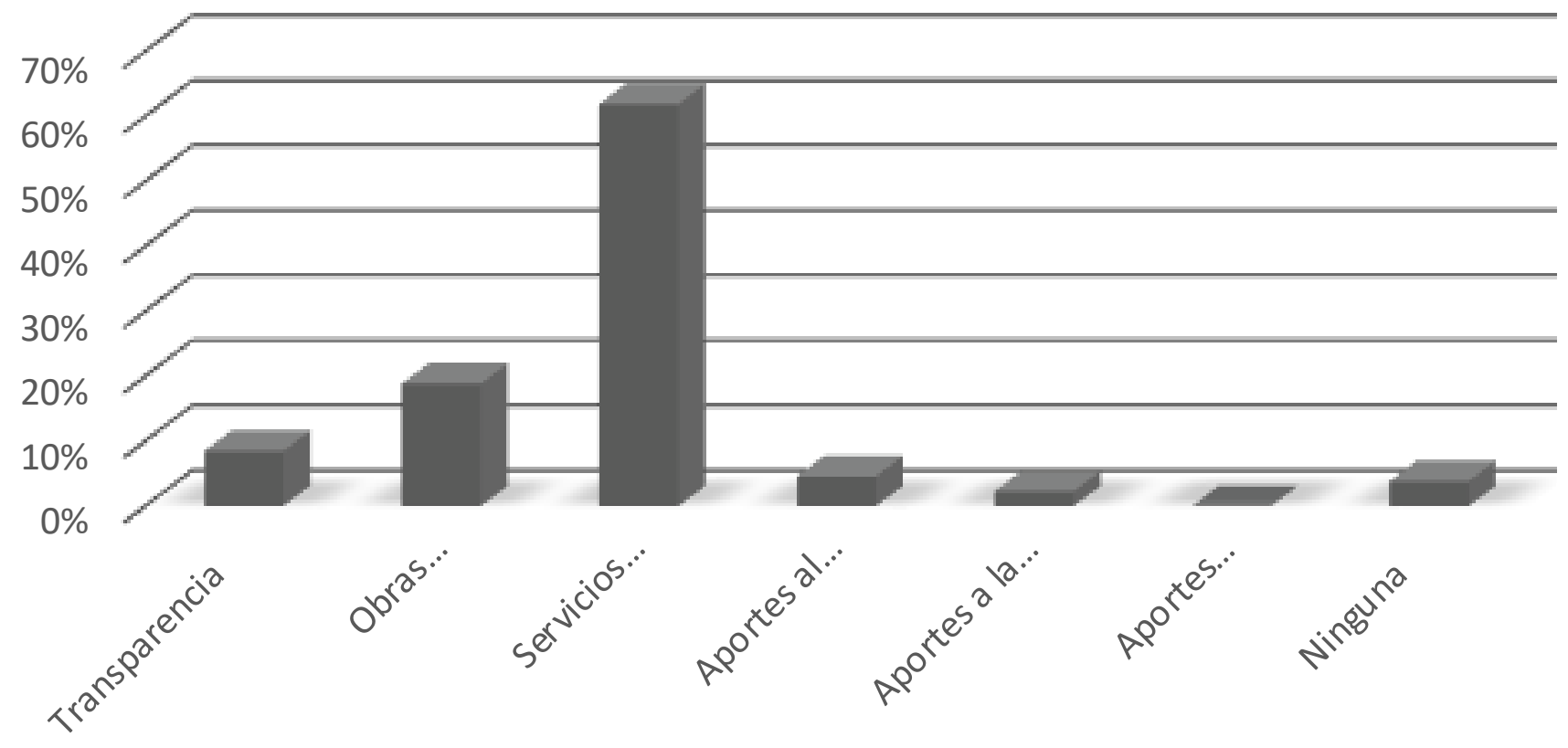

Figura 3. Porcentajes. Lo que mejor realiza la actual administración municipal

Fuente: tabla 3 de resultados de lo que mejor realiza la actual administración municipal.

96 | Ciencia y Sociedad 2020; 45(4, octubre-diciembre): 89-104 • Una Mirada desde lo Particular 


\section{Evaluación del ayuntamiento municipal mediante una encuesta de opinión en República Dominicana. \\ El caso del municipio de Baitoa en la provincia de Santiago}

Con esta pregunta se crea un contraste con relación a la pregunta anterior, esto permite tener una mejor interpretación de las observaciones generales del estudio. Según la tabla de resultados, el otorgamiento de los servicios básicos es de las acciones que mejor realiza la administración municipal que de mejor manera se valora entre la población de estudio, entre estos servicios se pueden enumerar la recogida de basura y la gestión de permisos para las construcciones de edificaciones, tal cual se expresaba a modo de ejemplo en los cuestionarios de la encuesta, siendo estas acciones valoradas de forma muy positiva en las secciones donde la población tiene más vocación urbana, como los es el centro urbano del municipio Baitoa.

Se entiende que la recogida de basura, por ejemplo, es un servicio fundamental en las funciones que realizan las alcaldías, pues es una acción vinculante a la buena gestión y como se observa es un elemento que pondera en gran alcance a la población, elementos logísticos para la recogida de basura relativas a los tiempos para la recogidas, los equipos utilizados para realizarlo y la disposición final de los desechos orgánicos e inorgánicos, todos ellos son elementos fundamentales que construyen una buena valoración de esta acción municipal.

De cada diez (10) ciudadanos, seis (6) entienden que los servicios municipales son la mayor fortaleza de la administración en sus dos primeros años. Lo cual es altamente ponderable, por entenderse que el municipio Baitoa a nivel geográfico es un territorio muy montañoso, esto representa un desafío por el nivel de dificultad que implica el recorrido de camiones colectores de desechos por todas las comunidades y su regreso al espacio de disposición final. Se advierte que la disposición final de los desechos sólidos debe mejorar en el municipio, la población no refleja de forma implícita por este elemento medioambiental tenga alta valoración por sus observaciones favorable a la recogida de basura a modo general.

Con el objetivo de determinar la percepción de cómo se trabaja la comunicación para informar a la población sobre el accionar de la administración con los recursos públicos, se preguntó: ¿Entiendes que la actual administración maneja e informa de manera correcta las acciones que realiza con los recursos que recibe del Estado y los árbitros?

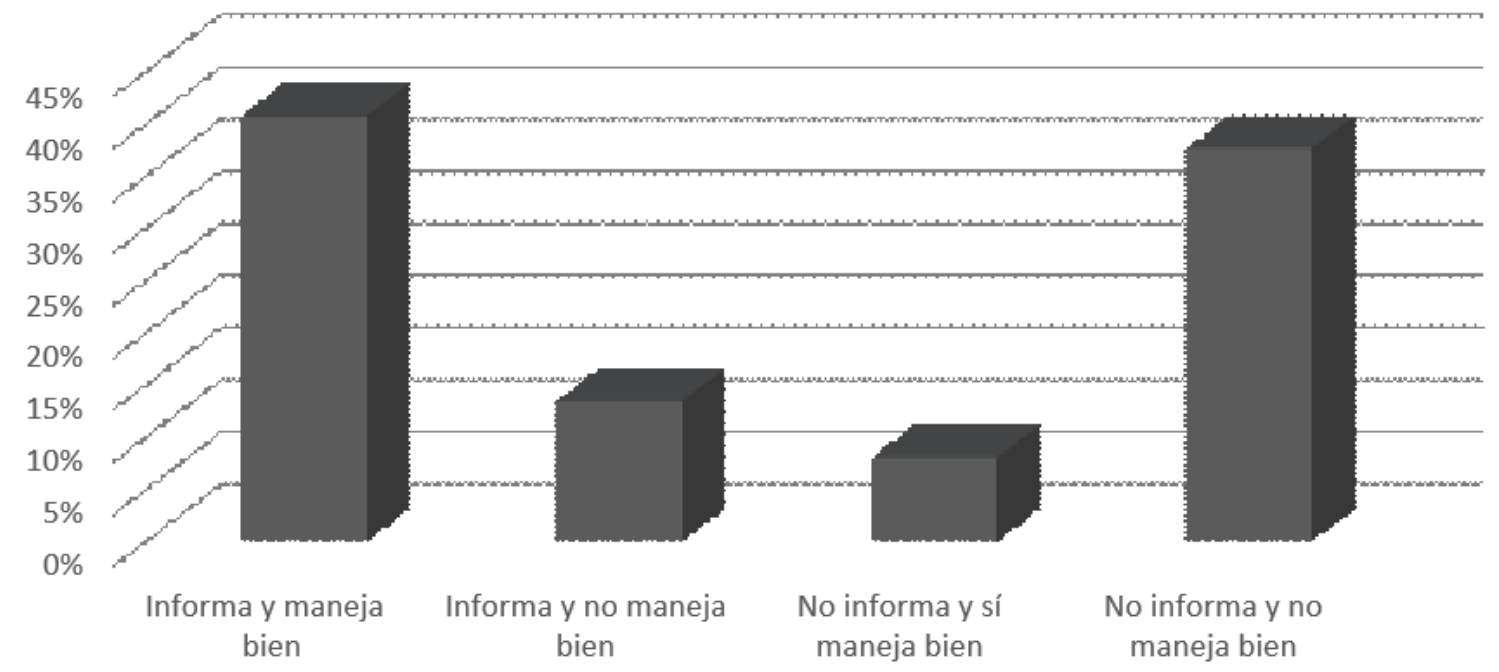

Figura 4. Porcentajes. El manejo y forma de informar de las acciones que realizan con los recursos que recibe del Estado y los árbitros la alcaldía

Fuente: tabla 4 de resultados alcanzados en la pregunta número 4 de este estudio. 
La población entiende que la administración informa bien y maneja bien sus recursos en un cuarenta y un por ciento (41\%), esto indica que ambos elementos se entrelazan el uno con el otro de forma armónica; sin embargo, en el desglose de la sumatoria del cincuenta y cuatro por ciento $(54 \%)$ que entiende que informa de manera correcta; un trece por ciento $(13 \%)$ entiende que no maneja bien los recursos, quedando en evidencia que informar bien no necesariamente está vinculado a que la población entiende que se manejen bien los recursos. Pero la misma pregunta muestra la lectura de un treinta y ocho por ciento $(38 \%)$ del total de los encuestados que concluyen que no informa ni maneja bien los recursos, para computar la conclusión de que un cincuenta y uno por ciento $(51 \%)$ entiende que no se manejan bien los recursos.

De este alto porcentaje que entienden que no se manejan bien los recursos, solo un trece por ciento $(13 \%)$ entiende que informan de sus ejecutorias de forma correcta. Los porcentajes de las personas que observaron que no se informa de manera correcta las acciones que se realizan en la alcaldía, también entienden que la gestión municipal no administra de forma correcta los recursos. La aparente deficiencia que tiene la alcaldía sobre su capacidad de informar las acciones que realizan con los recursos que perciben es evidente, cuando se observa que del cincuenta y cuatro por ciento (54\%) que percibe que se informa correctamente, valora en un cuarenta y un por ciento ( $41 \%$ ) el buen manejo de los recursos. Si el cuarenta y seis por ciento ( $46 \%$ ) de la población que dice que informan de manera inapropiada estuviera mejor informada de las acciones administrativas que realiza la alcaldía, entonces en un mayor porcentaje pudiera identificarse quienes entiendan que se hace un buen manejo de los recursos de la alcaldía.

Partiendo de que la percepción general de que la administración municipal es valorada positivamente, evidenciado en la pregunta número uno (1) donde marcó un sesenta y nueve por ciento $(69 \%)$ de valoración positiva, al contrastarlo con el treinta y ocho por ciento (38\%) de la población que entiende que no se maneja y administra bien la alcaldía, se evidencia que la valoración positiva de esta administración municipal no se sustenta necesariamente en la buena comunicación de cómo administra sus recursos; sino más bien, en la buena valoración que tiene la población de los servicios municipales que ofrece a la población.

Buscando conocer las expectativas de los parroquianos y determinar el nivel de confianza que tiene la población en las autoridades para la segunda mitad de la gestión municipal, se preguntó también: ¿Cuál es su expectativa para los próximos dos (2) años de gestión de la actual administración municipal del municipio Baitoa?

La población valora de forma especial la reparación de las vías de comunicación, sustentándose esto quizás en la realidad geográfica de las comunidades que conforman los focos poblacionales del municipio, los cuales en su mayoría son comunidades rurales por las que históricamente poder desplazarse por caminos de tierra en condiciones dignas es un elemento de alta valoración.

En comunidades como López, San José, Mocan y La Jagua, la reparación de caminos predominó numéricamente en las muestras. Es bueno señalar que estas comunidades nunca han tenido carreteras pavimentadas hasta la aplicación y realización de esta investigación y que por tradición la población del municipio Baitoa piensa (según la información obtenida a partir de entrevistas que se presentan más adelante) que las distintas administraciones municipales en la historia de la alcaldía de Baitoa no han tenido los recursos para pavimentar carreteras y caminos, pues se entiende que la alcaldía local solo pueden realizar pequeños tramos carreteros en concreto y tapar hoyos en la vía de principal acceso al municipio donde ya el gobierno central pavimentó hace muchos años, limitándose el gobierno municipal a trabajar de forma reaccionaria al mantenimiento improvisado y urgente. 


\section{Evaluación del ayuntamiento municipal mediante una encuesta de opinión en República Dominicana. \\ El caso del municipio de Baitoa en la provincia de Santiago}

Finalmente, se busca conocer cuál es la expectativa que tiene la población en la administración para los próximos ańos. Se formula la siguiente pregunta con el propósito de conocer cuál es la obra o acción municipal que más desean o esperan las distintas comunidades del municipio Baitoa: ¿Cuál entiendes es la acción o la obra municipal más importante que debería realizar la actual administración para mejorar su gestión municipal?

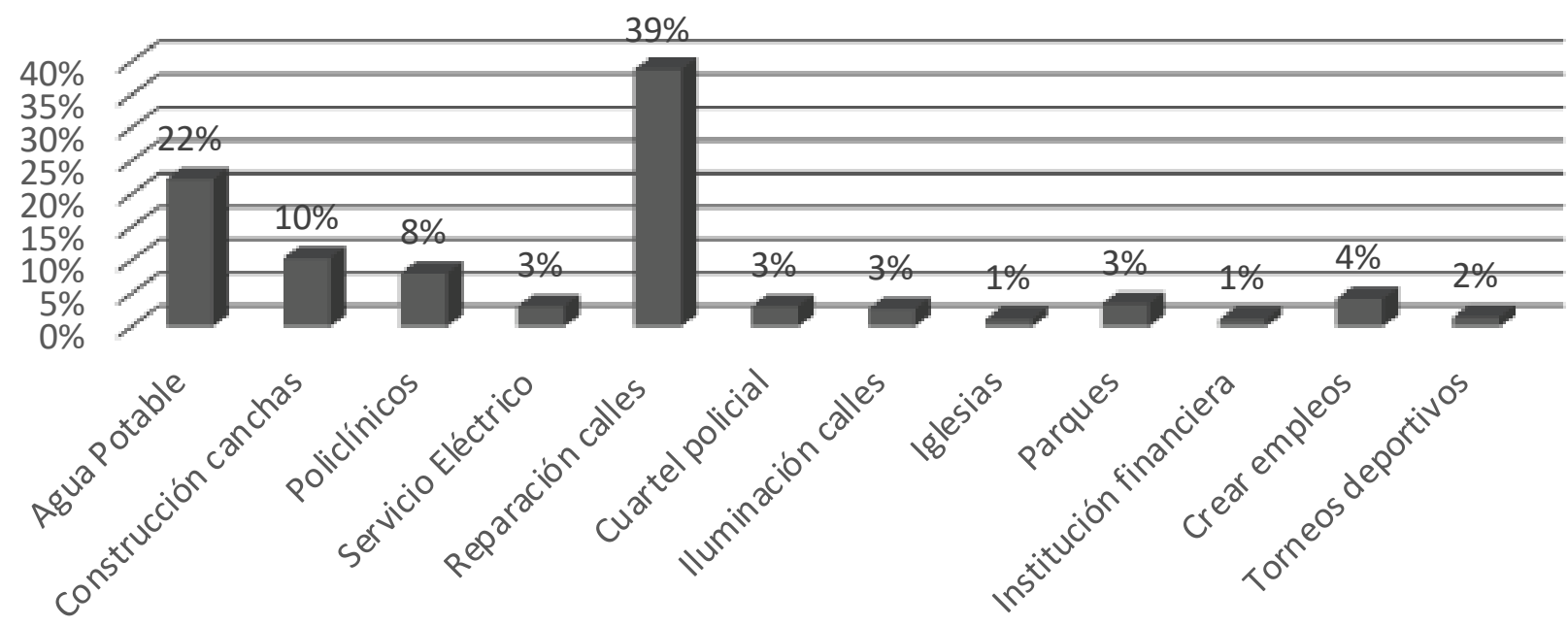

Figura 7. Porcentajes. Resultados sobre la acción o la obra municipal más importante que debería realizar la actual administración para mejorar su gestión municipal

Fuente: tabla 7 de resultados alcanzados en la pregunta número 7 de este estudio.

Con esta consulta se hace un aporte directo para mejorar la gobernanza del municipio y entender en dónde orbita la esperanza de la mayoría que entiende que la gestión municipal mejorará en los próximos años, según manifestaron en preguntas anteriores. Aquí se mantiene la tendencia de valorar las inversiones en infraestructuras, como lo indica la valoración mayoritaria a la reparación de las vías de comunicación terrestres mostrada en la pregunta número seis (6), en la que un cincuenta y siete por ciento $(57 \%)$ de la población valoró como lo más positivo las acciones realizadas en esa dirección.

En esta pregunta se pone de manifiesto el ciudadano que entendió que esa no fue la acción más importante realizada por la administración, pero que además entiende que deberían seguir siendo estas acciones las que se realicen con mayor frecuencia en los próximos dos ańos, por eso, cuando se consulta cuál es la acción que se debería realizar (planteando escenarios futuros), una vez más la reparación de vías de comunicación se destaca con el porcentaje mayoritario de un treinta y nueve por ciento (39 $\%)$ de la población encuestada.

Se entiende que en las respuestas recogidas en la pregunta número seis (6), existe gran valoración por las acciones realizadas para la mejora de caminos, carreteras y vías de comunicación terrestre en sentido general; sin embargo, en el levantamiento de la presente pregunta se determina que a pesar de ser bien valoradas esas acciones encaminadas a mejorar la infraestructura vial, el ciudadano entiende que estas deben incrementarse, lo que representa un desafío para las autoridades que buscan estar a nivel de las expectativas de mejoras en la administración municipal. Estas son acciones 
muy costosas en comparación con los recursos económicos que se destinan a otras demandas de la población, por lo que, al parecer, el presupuesto resulta insuficiente.

En un municipio de relieve montañoso y con población mayoritaria de un estilo de vida rural como lo es el municipio Baitoa, independientemente de que las autoridades municipales dicen que de manera constante están convocadas a reparar caminos vecinales, hasta el momento (de acuerdo con las entrevistas realizadas a expertos), no se hace un esfuerzo por adquirir maquinarias pesadas para mantenimiento de caminos, por entender que estas serían una carga muy pesada para el presupuesto que maneja la alcaldía.

La acción o la obra municipal más importante que debería realizar la actual administración para mejorar su gestión municipal, luego de la reparación o construcción de carreteras, es el abastecimiento de agua potable para toda su población, lo que se interpreta como la construcción del acueducto. En informaciones suministradas en las entrevistas; por más de 45 años, este municipio carece de un suministro constante de agua y de un servicio de agua potable de calidad, por ello, en la parte más urbana del municipio Baitoa, se destaca esta obra a futuro con valoración de un sesenta por ciento $(60 \%)$. Hasta el momento, no se conoce experiencia alguna de que una alcaldía haya construido un acueducto para todos sus habitantes en la región norte de la República Dominicana, de ahí su originalidad y pertinencia.

Destaca que la construcción de iglesias, canchas y parques tengan mención por los encuestados en esta pregunta abierta, así como también destacamentos policiales y policlínicas dentro del renglón de construcción de infraestructuras, sumando entre todos ellos una expectativa para el rubro de construcción de infraestructuras de un ochenta y siete por ciento $(87 \%)$. La iluminación de caminos y la realización de torneos deportivos marcan un cinco por ciento $(5 \%)$ de expectativa en inversiones en esa dirección y, finalmente, dentro del renglón de lo que se entiende debe ser gestionado por las autoridades municipales en los próximos dos años, está la mejora del servicio eléctrico que proporciona el Estado, la creación de empleos y gestionar la llegada de instituciones financieras al municipio, sumando todo ello el restante ocho por ciento (8 $\%)$ de las expectativas de los habitantes del municipio Baitoa.

\section{Conclusiones}

La encuesta de opinión implementada en el Municipio Baitoa arroja resultados interesantes sobre la percepción de la ciudadanía sobre el gobierno del ayuntamiento municipal de esta localidad, electo para el periodo electoral 2016-2020. Considerando los dos primeros años de gobierno, los resultados indican que el $69 \%$ percibe de manera buena o excelente la actual gestión municipal. La población valora mayoritariamente la reparación de caminos y carreteras, de forma particular en los sectores que carecen de carreteras pavimentadas o en las que la pavimentación existente presenta un avanzado nivel de deterioro. Se hace referencia a "reparación" y no a construcción porque la población parece entender que los presupuestos municipales no son suficientes para construir carreteras pavimentadas, sin embargo, valoran los esfuerzos de acondicionarlas y reparar pequeños tramos muy deteriorados.

En los aspectos de valoración están los servicios que se ofrecen a la población como lo es la recogida de basura y la construcción de obras municipales, dejando en un segundo plano los aspectos relativos a la inversión en cultura, deportes y aportes sociales. Sin embargo, la poca construcción de obras municipales es el principal sustento de percepción de quienes valoran negativamente la alcaldía, entendiendo que obras municipales 


\section{Evaluación del ayuntamiento municipal mediante una encuesta de opinión en República Dominicana. \\ El caso del municipio de Baitoa en la provincia de Santiago}

podrían ser la construcción de centros comunales, parques, aceras, locales, plazas y otras obras construidas con recursos propios de la alcaldía.

Las alcaldías de comunidades rurales en la República Dominicana no presentan antecedentes significativos en realizar esfuerzos por adquirir maquinarias de movimiento de tierra para responder a estas constantes demandas, limitando sus inversiones en maquinarias a la compra de camiones que adaptan a los servicios de recogida de basura, partiendo del razonamiento de no querer comprometer los bajos ingresos mensuales de las alcaldías al pago de estos posibles costosos equipos pesados, por lo que se ven obligados a alquilarlos constantemente, en especial luego de las temporadas lluviosas del Caribe.

La capacidad de informar a la población de las acciones realizadas en las alcaldías, plantea un empuje importante para la buena percepción en el manejo de los recursos y de la administración municipal en sentido general. Independientemente de que existen cúmulos significativos de ciudadanos que valoran la comunicación y el manejo de los recursos municipales de forma no positiva, es ponderable la relación que existe entre los porcentajes de las personas que observaron que no se informa de manera correcta las acciones municipales con los que entienden que la gestión municipal no administra de forma correcta los recursos, planteando un posible vínculo entre no informar bien y crear una percepción negativa.

Se detectan elementos importantes a mejorar en la administración municipal como lo es la trasparencia en el manejo de los recursos, la planificación y la participación e integración de todos los sectores que conforman la fuerza viva de las comunidades, sin embargo, la población valora positivamente la gestión teniendo confianza en que mejorará los escollos negativos reconocidos por las autoridades, los munícipes y los expertos; pero será importante que la comunicación sea aún más efectiva con la población, pues no solo permite mantener la percepción positiva en sentido general, sino a mejorar la confianza en que la administración mejorará en los próximos dos años.

La buena valoración de los servicios básicos ofrecidos por la administración, además de la comunicación efectiva de las autoridades sobre la escasez de recursos, parecen ser asimilados de forma positiva por la población, reflejando una alta valoración en las propias comunidades que demanda inversión en obras municipales, por lo que se recomienda que las autoridades mejoren y amplíen sus capacidades comunicativas con la población.

El presupuesto aprobado para el Municipio Baitoa está sustentado en el modelo de presupuesto participativo, el cual convoca a las comunidades en asambleas para que las comunidades propongan a las autoridades las obras o acciones municipales más necesarias en sus demarcaciones, dándole a la administración ideas de cómo responder a la población con inversiones mínimas y formas de alimentar la distribución de fondos para la realización de obras municipales a partir de las cargas poblacional de cada sección del municipio, esta acción es un elemento que alimenta la buena percepción de las autoridades ante sus gobernados.

En las secciones del municipio donde la administración es altamente valorada, la población celebra los buenos servicios municipales ofrecidos, pero también entiende que la inversión en vías de comunicación terrestre es mínima, por lo que se recomienda la estructuración de un plan de reparación y pavimentación de los caminos y carreteras del municipio, así como también la adquisición de equipo de movimiento de tierra para el acondicionamiento de los caminos en los sectores montańosos donde no es posible llevar el plan de pavimentación o recuperación de tramos de camino en muy mal estado con depósito de concreto. 
La población dominicana es mayoritariamente joven, y como jóvenes que habitan el Caribe las actividades deportivas están presente el año completo por las condiciones climatológicas favorables, es posible que a esta característica se deba el hecho de que la población valora más los aportes al sector deportivo. Por otra parte, preocupa la casi nula percepción de valoración al sector cultural, puesto que la identidad municipal está vinculada a la realización de festivales culturales como las fiestas patronales, los encuentros juveniles teatrales, la existencia de museos históricos, la creación de una banda municipal musical, la realización de festivales de canto; acciones que son mínimas en el municipio y otras inexistentes, por lo que se recomienda la valoración e inversión en aspectos culturales desde la alcaldía.

Los anhelos de la población expresados en las preguntas abiertas hacia el futuro continúan encaminados a la mejora en el desplazamiento vial, así como la factibilidad de mejorar los servicios de distribución de agua potable con mayor énfasis en los cascos urbanos, lo cual es un elementos más vinculante al gobierno central por los grandes recurso económicos que conllevaría lograr este anhelo de la población, pero la población genera expectativas de que la alcaldía pueda alivianar la carga social de la falta de agua, con la distribución del líquido dentro de los programas de ayudas sociales o liderar algún proyecto social comunitario que pueda colectar las aguas de las fuentes acuíferas que bordean este municipio, encaminándolas hacia los hogares.

Se recomienda a los ciudadanos manifestar sus opiniones en las asambleas convocadas por la sala capitular para exponer sus preocupaciones, necesidades o evaluaciones a las políticas municipales, buscar y evaluar la información contenida en informes y en los presupuestos anuales, como también en las llamadas partidas participativas asignadas en el presupuesto.
En este estudio se evidencia que la buena gobernanza municipal puede seguir mejorando a partir de las capacidades que tengan las autoridades de mejorar la comunicación con sus ciudadanos, así como también la de responder a su esperanza de mejoras en los sistemas de comunicación vía la realización de más obras municipales. Resulta prioritario el apoyo al deporte y a la cultura y otras acciones encaminadas a mejorar la trasparencia en la gestión municipal.

La construcción de obras municipales y comunitarias con la construcción o reparación de canchas deportivas, la reparación de caminos y carreteras de zonas montańosas y las carreteras troncales cercanas al centro urbano del municipio, la reparación de aceras, contenles y construcción de sistemas de drenajes de aguas pluviales, así como también la construcción de zonas de esparcimiento e interacción social como parques y plazoletas son fundamentales, para que la población pueda percibir que los fondos públicos que manejan las autoridades son invertidos de forma correcta, en obras que mejoran su calidad de vida.

El manejo de la comunicación institucional del ayuntamiento con sus gobernados debe mejorar significativamente, se recomienda realizar esfuerzos en constituir un equipo de relaciones públicas y disponer de recursos para su capacitación, asegurando que informen a la comunidad de las ejecutorias que realiza o piensa realizar el ayuntamiento en materia de inversión municipal, el tiempo para su ejecución, concientizar sobre la necesidad de la misma explicando los propósitos y los objetivos a corto y largo plazo por los medios tradicionales impresos y los nuevos medios digitales, como las redes sociales; así como también por una página web destinada a cumplir con las exigencias de trasparencia exigidas en recientes legislaciones del país, tomando en cuenta que la misma deberá ser actualizada de forma constante y precisa. 


\section{Referencias}

Sojo, I. A. (1999). El valor de las encuestas de opinión pública. Revista Latina de Comunicación Social 2(15), 1-9.

Böhm, L., P. Márquez, J. Poblette, M. Repossi, y R. Reta (2007) Manual de Planificación Estratégica Municipal. Buenos Aires: Konrad-Adenauer-Stiftung.

Cabrera, L. (2001). Efectos de la impunidad en el sentido de justicia. Psicología politica (23), $37-58$.

Candia-Falcón, G. (2017). El estado de derecho: ¿al servicio de los derechos fundamentales?, Revista de Derecho, 30(2), 181-201.

Canto-Sáenz, R. (2017). Participación ciudadana, pluralismo y democracia. TLA-MELAUA. Revista de Ciencias Sociales, 10(41), 54-75.

Casas-Anguita J., J.R. Labradora., \& J.D. Campos (2003). La encuesta como técnica de investigación. Elaboración de cuestionarios y tratamiento estadístico de los datos (I), Aten Primaria, 31(8), 527-538.

Cogco-Calderón, A. R., M. Rodríguez-Vargas, \& J.A. Pérez-Cruz (2010). Un análisis de la política social en México a través de los programas implementados por La secretaría de desarrollo social y su relación con la descentralización de funciones: una mirada desde lo local. Ibero fórum. Revista de Ciencias Sociales de la Universidad Iberoamericana, 5(9), $1-35$.

Fernández Ruiz, J. (2015). El legado de George Gallup. Ciencia juridica, 4(7), 37-54.

Gallup, G., \& Forbes, S. (1940). The Pulse of Democracy: The Public Opinion Polls and How It Works. New York: Ed. Simon and Shuster.

Gimeno-Feliu, J.M. (2019). Corrupción y seguridad. La necesidad de un marco normativo de las decisiones públicas anclado en los principios de integridad y de transparencia”, R.I.T.I. Revista Internacional de Transparencia e Integridad, (9), 1-17.
Gómez MacFarland, C. A. (2017). Los planes de desarrollo municipal en México y la participación ciudadana. Un análisis del marco jurídico. Boletín mexicano de derecho comparado, 50(150), 1149-1177.

González Ibarra, J. R; Tapia Vega, B. \& Valencia, A. (Eds.). (2017). Derecho y buen gobierno. México DF: Cámara de Diputados, LXIII Legislatura.

González-Murillo, H. (2003). La planificación y gestión del desarrollo territorial en municipios con dificultades de gobernabilidad. Revista Opera, 4(4), 37-78.

Gutiérrez, E. (2018). Corrupción pública: concepto y mediciones. Hacia el Public compliance como herramienta de prevención de riesgos penales, Revista Politica Criminal, 13, 104-143.

Hernández-Sampieri, R.; Fernández-Collado, C \& Pilar Baptista-Lucio, M. (2014). Metodología de la Investigación, Sexta Edición. México D.F: Ed. McGraw Hill.

Jiménez-Polanco, J. (2016). La corrupción política en la República Dominicana y la entronización del partido cartel, RIPS. Revista de Investigaciones Politicas y Sociológicas, 15(2), 9-27.

Kavanagh, D. (1994). Las Encuestas de Opinión Pública. Estudios Políticos, (53), 145-169.

Kuschick, M. (2006). Encuestas de Opinión, imagen y cambio de actitud, RMCPS. Revista Mexicana de Ciencias Politicas y Sociales, 48(196), 143-157.

Kurschick, M. (2004). Teorías del comportamiento electoral y algunas de sus aplicaciones, RMCPS. Revista Mexicana de Ciencias Politicas y Sociales, 46(190), 47-70.

Lippmann, W. (1964). La Opinión Pública. Buenos Aires: Editorial Fabril.

Luna, J.P., y H. D. Soifer (2015). Perspectivas desde el Barómetro de las Américas: 2015 Instituto de Ciencia Politica-Pontificia Universidad Católica de Chile y Departamento de Ciencia Politica-Temple University, Número 119. 
Mañas-Ramírez, B. (2005). Los Orígenes Estadísticos de las Encuestas de Opinión, EMPIRIA. Revista de Metodología de Ciencias Sociales, (9), 89-113.

Martínez de Luna, I. (2008). Encuesta de Opinión: de la Teoría a la Practica. Metodología de Encuestas, (10), 7-25.

Molina-Molina, J., \& Mayor Balsas, J.M (2018). Gobierno Abierto, Transparencia y ética Pública, R.I.T.I. Revista Internacional de Transparencia e Integridad, (8), pp. 1-10.

Monzón-Arribas, C. (1990). La Opinión Pública: Teorias, concepto y métodos. Madrid: Editorial Tecnos.

Orduña-Trujillo, E.L. (2006). Derechos humanos y credibilidad política. Boletín mexicano de derecho comparado, 39(116), 481-499.

Peter, j., Ed Fieldhouse y Hanhua Liu (2011). How civic is the civic culture? Mapping the routes to community participation using 2005 English Citizenship Survey. Political Studies, 59(2), 230-252.

República Dominicana, Congreso Dominicano (2017). Ley 176-07 del Distrito Nacional y los Municipios de la República Dominicana (versión electrónica) publicado por la gaceta oficial del estado dominicano el 2017. Recuperado el 29 de julio del 2018 de http:// www.icpard.org/media/15836/A5S78_ Ley__176-07_de_Ayuntamiento_y_Municipios.pdf
República Dominicana, Congreso Dominicano (2013). Ley 69-13 que crea el Municipio Baitoa, Santo Domingo de Guzmán (Versión electrónica) publicado por la gaceta oficial del estado dominicano el 2014. Recuperado el 01 de agosto del 2018 de https://baitoerosoy. blogspot.com/2013/06/municipio-baitoa-promulgado-en-la-ley.html

SAGARPA-INCA (2004). Guía para el Buen Gobierno Municipal. Introducción al Gobierno y Administración Municipal, (Tomo No. 6). México DF: Secretaría de Agricultura, Ganadería, Desarrollo Rural, Pesca y Alimentación-Instituto Nacional para el Desarrollo de Capacidades del Sector Rural.

Schneider, C.Y.W. (2011). ¿Transformación democrática o control político? Análisis comparado de la participación ciudadana institucional en América del Sur. Ícono. Revista de Ciencias Sociales, (40), 21-39.

Serrano-Rodríguez, A. (2015). La participación ciudadana en México. Estudios Políticos, 9(34), 93-116.

Sorribas, P., \& Garay-Reyna, Z. (2014). La participación, entre la democracia participativa y la democracia directa. Aportes desde un enfoque psicosocial. POLIS, 10(2), 39-69.

Sunkel, G. (1989). Las encuestas de opinión pública: entre el saber y el poder (No. 439). Santiago de Chile: Facultad Latinoamericana de Ciencias Sociales.

\section{Datos de filiación}

Leonardo Pérez. Candidato a Doctor en Proyectos por la Universidad Internacional Iberoamericana (UNINI, México). Master en Proyect Management (Internacional) por la Universidad Internacional Iberoamericana (UNINI, Puerto Rico). Ingeniero Industrial por la Universidad Autónoma de Santo Domingo (Santiago, DO).

Marco Antonio Rojo Gutiérrez. Doctor en Estudios Sociales (Summa Cum Laude) por la Universidad Autónoma Metropolitana (UAM, México). Maestro en Estudios Sociales, en la línea de Investigación de Economía Social por la Universidad Autónoma Metropolitana (UAM, México). Licenciado en Economía por la Universidad Autónoma Metropolitana (UAM, México). Pertenece al Sistema Nacional de Investigadores del Consejo Nacional de Ciencia y Tecnología (SNI-CONACYT, México). Actualmente se desempeña como Profesor-Investigador de Tiempo Completo en la Universidad Internacional Iberoamericana (UNINI, México). 